\title{
Magnetization Reorientation in Self-Organized Fe Nanowire Arrays
}

\author{
N. Rougemaille and A.K. Schmid
}

Lawrence Berkeley National Laboratory, 1 Cyclotron Road MS/72, Berkeley, CA 94720

Using Spin-Polarized Low-Energy Electron Microscopy (SPLEEM), we study the assembly of iron nanowires on a (110) tungsten surface and we investigate correlations between self-organization mechanisms and magnetic domain microstructures. SPLEEM is an electron microscopy technique based on the fact that the reflectivity of a magnetic surface for a spin-polarized low-energy electron beam depends on the relative orientation of the magnetization and the incoming spin polarization. In SPLEEM, the spin-polarization of the electron beam can be oriented in all spatial directions, thus allowing the determination of unknown magnetic domain microstructures. Combining real-time imaging capability and good spatial resolution with very good magnetic sensitivity, SPLEEM is a uniquely suitable tool for quantitative characterization of magnetic configurations and structureproperty relationships.

Starting from essentially flat nanometer-thick iron films, epitaxially grown at room temperature on a (110) tungsten single crystal, we used carefully tuned annealing schedules to produce periodic arrays of submicron / nanoscale ferromagnetic wires. The wires are elongated along the [001] direction and a wetting layer covers the tungsten surface between the wires. The structural transition from continuous film to nanowires is accompanied with an in-plane 90 degrees rotation of the magnetic easy-axis $[1,2]$. While the magnetization of the continuous iron film initially lies along the [1-10] direction due to the strong anisotropy of the tungsten surface [3], it becomes aligned in the [001] direction as the nanowire arrays grow during annealing [1,2]. Different factors have been proposed to contribute as driving mechanism for the rotation of the magnetic easy axis after annealing: reduction of the magnetoelastic anisotropy at the $\mathrm{Fe} / \mathrm{W}$ interface [2], reduction of the surface anisotropy in the nanowires [1], and contribution of the shape anisotropy [2].

By mapping the local magnetization directions in real-time, we study the role of the kinetics of the structural transition on the magnetization reversal process. For low annealing temperatures, remarkably regular periodic arrays of iron wires can be prepared (fig.1.a). The wires keep the magnetization of the continuous film until the shape anisotropy becomes the dominant magnetic anisotropy (fig.1.b and 1.c). Before the magnetization reversal is complete, the wires are found to be in an intermediate state where the magnetization exhibits Landau domains. However, the shapes of the wires, such as width, length and distance between the wires, are strongly influenced by the annealing temperature. While low annealing temperatures favor the nucleation of well-defined straight de-wetting zones which do not affect the magnetization distribution of the continuous film, high annealing temperatures lead to the formation of rounded-shape de-wetting zones where the magnetization reversal takes place (fig.2). Our findings thus highlight how the relative importance of the different factors contributing to the magnetic reorientation (listed above) can be influenced by controlling the kinetics of the process.

References

[1] D. Sander et al., J. Phys. D: Appl. Phys. 31 (1998) 663.

[2] L. Lu et al., J. Phys.: Condens. Matter 10 (1998) 2873. 
[3] U. Gradmann et al., Appl. Phys. A 39919860101.

[4] This work was supported by the Délégation Générale pour l'Armement (contract 9860830051).
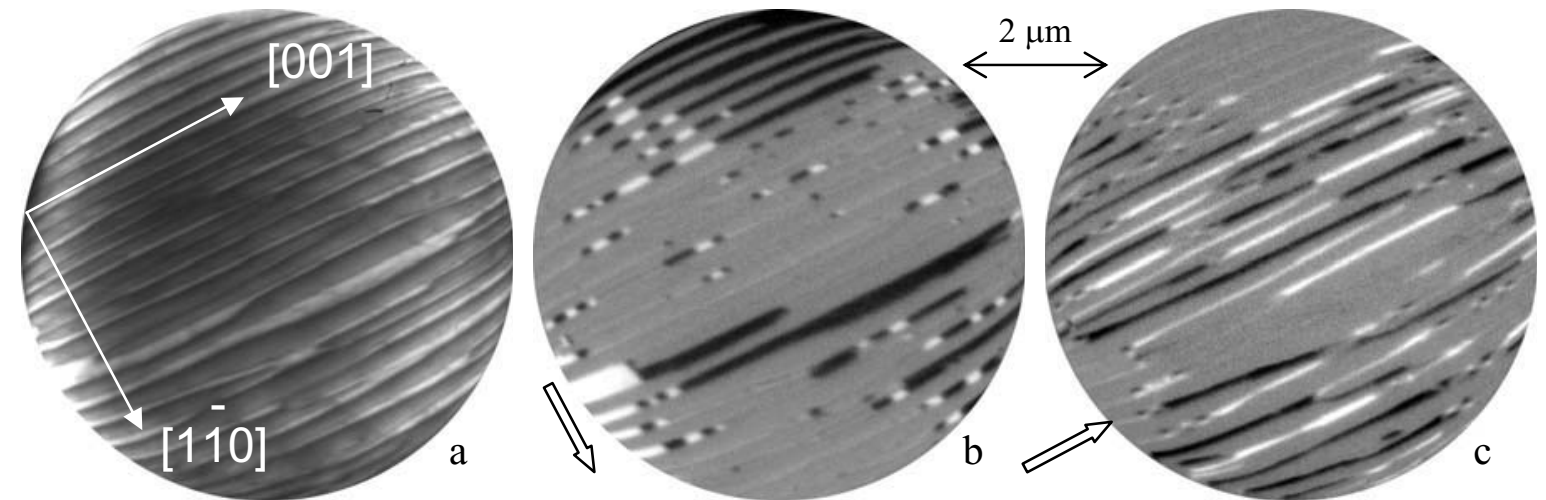

FIG. 1. Periodic array of magnetic wires. Topographic (a) and magnetic (b and c) images of the surface after annealing (at $325^{\circ} \mathrm{C}$ for 10 hours) a room-temperature-grown 14 monolayer-thick iron film. The 2 thick white arrows indicate the incident spin polarization. A black (white) contrast means that the local magnetization vector has a component parallel (antiparallel) to the incoming spins. For the thickest wires, the surface anisotropy is strong enough to keep the magnetization along the [1-10] direction, while the magnetization of the thinnest wires has rotated due to shape anisotropy. Landau domains initiate the magnetization reorientation when wires are approximately $100 \mathrm{~nm}$ wide.

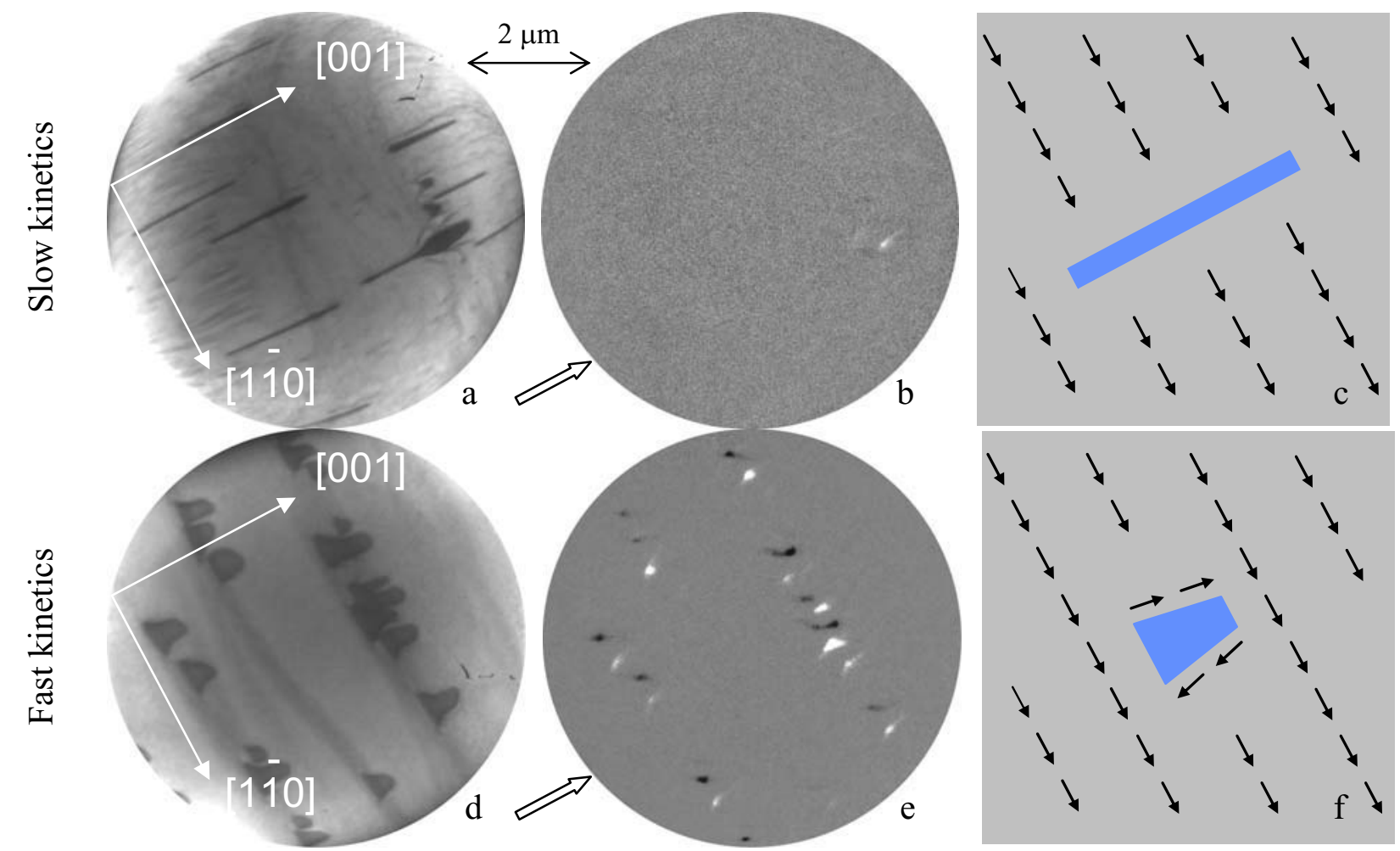

FIG. 2. Role of the kinetics on de-wetting and magnetization reversal processes. De-wetting zones appear in dark in the 2 topographic images (a, d). The corresponding magnetic images (b, e), and their associated sketches (c, f) which represent the local magnetization (black arrows) near a dewetted region (in blue), show how the kinetics affects the magnetization distribution of the film. 